Pacific Journal of Mathematics

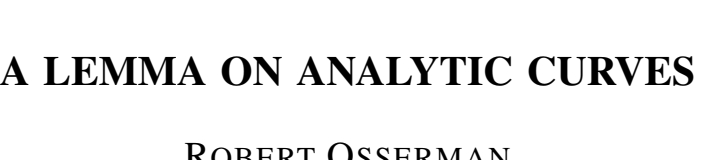




\section{A LEMMA ON ANALYTIC CURVES}

\section{ROBERT OSSERMAN}

The following lemma turns out to be useful in many places in Riemann surface theory. It is generally sufficient to have piecewise analyticity, rather than analyticity, but the availability of analytic curves will invariably make proofs simpler. This is especially true of the second half of the lemma, since an analytic Jordan curve on a Riemann surface is defined by a mapping of the unit circle into the surface and this mapping can be extended to a one-to-one map of an annulus into the surface. The extended mapping can be used, for example, to define explicitly differentials on the surface having prescribed properties.

The proof which we give is perhaps not the most straightforward one but has certain advantages over the usual type of reasoning involving subdivision of the parameter interval.

Lemma. Every closed curve on a Riemann surface is homotopic to an analytic closed curve, and homologous to a finite sum of analytic Jordan curves.

REMARK. By homology we mean singular homology. We are not concerned here with the choice of definition since we only use the following two properties of singular homology.

(1) Homotopic curves are homologous.

(2) If a closed curve $C$ is defined by a mapping $f(t)$ on the interval $I$ and if there is a subdivision of $I$ into intervals $I_{k}, k=1, \cdots, n$, and if the restriction of $f(t)$ to $I_{k}$ defines a closed curve $C_{k}$, then $C$ is homologous to $\sum_{k=1}^{n} C_{k}$.

We may also word the lemma as follows:

(a) Every homotopy class on a Riemann surface contains an analytic curve.

(b) Every singular homology class on a Riemann surface contains a cycle of the form $\sum_{k=1}^{n} C_{k}$ where the $C_{k}$ are analytic Jordan curves.

Proof. Let $R$ be the Riemann surface and $C$ an arbitrary closed curve. Let $R^{*}$ be the universal covering surface of $R$ and let $p^{*}$ be a point on $R^{*}$ which projects onto a point $p$ of the curve $C$. If we continue along the curve $C$ starting at $p^{*}$ we arrive at a point $q^{*}$ which also projects onto $p$. Any arc joining $p^{*}$ to $q^{*}$ will, by definition of the universal covering surface, project onto a curve homotopic to $C$. In

Received July 15, 1958. This paper was written while the author was a Temporary member of the Institute of Mathematical Sciences at New York University. 
particular, an analytic arc will project onto a curve which is analytic except possibly at $p$. To prove part (a) of the lemma we make a simple choice of arc which will give a curve analytic also at $p$.

We note first that if $p^{*}$ and $q^{*}$ coincide, then $C$ is homotopic to zero, and any small circle through $p$ will be homotopic (and homo logous) to zero, hence to $C$. In particular if $R^{*}$ is the sphere, then $R$ is itself the sphere and $C$ must have been homotopic to zero.

The other two possibilities are that $R^{*}$ is the plane or the interior of the unit circle. In the first case the cover transformation taking $p^{*}$ into $q^{*}$ is a linear transformation which takes the line through $p^{*}$ and $q^{*}$ into itself. The projection of the straight line segment joining $p^{*}$ to $q^{*}$ will therefore be analytic even at $p$.

If $R^{*}$ is the interior of the unit circle, then the cover transformation taking $p^{*}$ into $q^{*}$ will be a linear fractional transformation with either one or two fixed points on the unit circle. The circle through $p^{*}$ and $q^{*}$ and the fixed point (or points) will be mapped onto itself, so that again the arc of this circle joining $p^{*}$ to $q^{*}$ will project onto an analytic curve homotopic to $C$.

This proves part (a) of the lemma.

We have obtained in particular a simple proof that every closed curve is homologous to an analytic closed curve, which is in itself a useful fact.

The proof of part (b) requires a bit more effort. The case where $R^{*}$ is the plane is almost trivial since (except where $R$ is itself the plane, hence simply connected) the group of cover transformations is either of the form $z+n b$ where $b$ is a fixed complex number and $n$ runs through the integers, or else of the form $z+m a+n b$ where $a$ and $b$ are fixed and $m$ and $n$ run through the integers. In the first case $p^{*}$ and $q^{*}$ will correspond to a pair of points of the form $z_{0}$ and $z_{0}+n b$, so that the projection of the segment from $z_{0}$ to $z_{0}+n b$ will consist of the projection $C^{\prime}$ of the segment from $z_{0}$ to $z_{0}+b$ covered $n$ times. Hence $C$ is homologous to $n C^{\prime}$. But $C^{\prime}$ is a Jordan curve since no two points on the segment between $z_{0}$ and $z_{0}+b$ are equivalent.

Similarly, in the second case if $p^{*}$ and $q^{*}$ correspond to $z_{0}$ and $z_{0}+m a+n b$, we simply take the straight line segment from $z_{0}$ to $z_{0}+m a$ followed by the segment from $z_{0}+m a$ to $z_{0}+m a+n b$.

Finally, in the case where $R^{*}$ is the interior of the unit circle, we consider the metric fundamental polygon $P$ consisting of all points in the unit circle which are nearer (in the non-euclidean metric) to the point $p^{*}$ than to any point equivalent to $p^{*}$ under a cover transformation ${ }^{1}$. If $T$ is the cover transformation which takes $p^{*}$ into $q^{*}$ we may represent

1 A simplification in the proof at this point is due to the referee.

All the basic information about the fundamental polygon can be found, for example, in the book of Nevanlinna, Uniformisierung, Chapter VII, and in particular, \$7.15. 
it as $T=\prod_{k=1}^{n} T_{k}$, where each $T_{k}$ is a cover transformation taking one side $a_{k}$ of $P$ onto another side $b_{k}$. The sides $a_{k}$ and $b_{k}$ are circular arcs, and we can form the Riemann surface $R_{k}$ which consists of the interior points of $P$ together with inner points of the $\operatorname{arcs} a_{k}$ and $b_{k}$ with equivalent points under $T_{k}$ identified. Then $R_{k}$ is doubly-connected and may be mapped conformally onto an annulus $r_{1}<|w|<r_{2}$, where $r_{1}=0$ if $a_{k}$ and $b_{k}$ have a vertex in common. This annulus is one-to-one conformally equivalent to $R_{k}$ and hence to the surface $R$ cut along certain analytic arcs. Let $C_{k}$ be the curve in $R$ corresponding to that circle $|w|=r^{\prime}$ which passes through the image of the point $p$. Then $C_{k}$ is a Jordan curve passing through $p$, and if we continue $R^{*}$ along $C_{k}$ starting at a point $p_{k}{ }^{*}$ of $a_{k}$, we arrive at the point $q_{k}{ }^{*}$ of $b_{k}$ which is the image of $p_{k}{ }^{*}$ under $T_{k}$. Hence the curve $C_{k}$ generates the transformation $T_{k}$, and the curve $C^{\prime}=\prod_{k=1}^{n} C_{k}$ is a closed curve at $p$ which generates the transformation $T$. Thus $C^{\prime}$ is homotopic to $C$, and using the properties of singular homology remarked above we have that $C$ is homologous to $C^{\prime}$ which is homologous to $\sum_{k=1}^{n} C_{k}$, proving the lemma.

STANFORD UNIVERSITY. 



\section{PACIFIC JOURNAL OF MATHEMATICS}

\section{EDITORS}

\section{David Gilbarg}

Stanford University

Stanford, California

R. A. Beaumont

University of Washington

Seattle 5 , Washington
A. L. Whiteman

University of Southern California

Los Angeles 7, California

L. J. PAIGE

University of California

Los Angeles 24, California

\section{ASSOCIATE EDITORS}
E. F. BECKENBACH
C. E. BURGESS
E. HEWITT
A. HORN

\author{
V. GANAPATHY IYER \\ R. D. JAMES \\ M. S. KNEBELMAN \\ L. NACHBIN
}
I. NIVEN
E. G. STRAUS
T. G. OSTROM
H. L. ROYDEN
G. SZEKERES
M. M. SCHIFFER
F. WOLF
K. YOSIDA

\section{SUPPORTING INSTITUTIONS}

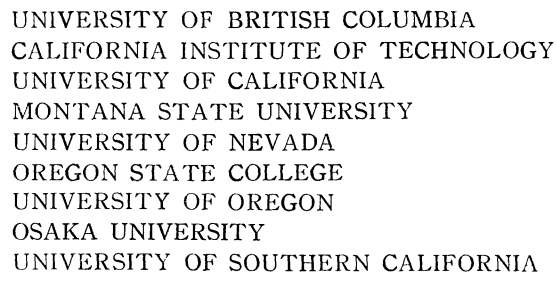

UNIVERSITY OF BRITISH COLUMBIA CALIFORNIA INSTITUTE OF TECHNOLOGY UNIVERSITY OF CALIFORNIA MONTANA STATE UNIVERSITY UNIVERSITY OF NEVADA OREGON STATE COLLEGE UNIVERSITY OF OREGON OSAKA UNIVERSITY UNIVERSITY OF SOUTHERN CALIFORNIA

STANFORD UNIVERSITY

UNIVERSITY OF TOKYO

UNIVERSITY OF UTAH

WASHINGTON STATE COLLEGE

UNIVERSITY OF WASHINGTON

AMERICAN MATHEMATICAL SOCIETY CALIFORNIA RESEARCH CORPORATION HUGHES AIRCRAFT COMPANY SPACE TECHNOLOGY LABORATORIES

Printed in Japan by Kokusai Bunken Insatsusha (International Academic Printing Co., Ltd.), Tokyo, Japan 


\section{Pacific Journal of Mathematics}

\section{Vol. 9, No. 1 \\ May, 1959}

Julius Rubin Blum and Murray Rosenblatt, On the structure of infinitely

divisible distributions . ............................. 1

Robert Geroge Buschman, Asymptotic expressions for

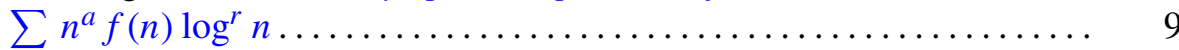

Eckford Cohen, A class of residue systems $(\bmod r)$ and related arithmetical

functions. I. A generalization of Möbius inversion .............. 13

Paul F. Conrad, Non-abelian ordered groups ................... 25

Richard Henry Crowell, On the van Kampen theorem............... 43

Irving Leonard Glicksberg, Convolution semigroups of measures ........ 51

Seymour Goldberg, Linear operators and their conjugates ............ 69

Olof Hanner, Mean play of sums of positional games .............. 81

Erhard Heinz, On one-to-one harmonic mappings ................ 101

John Rolfe Isbell, On finite-dimensional uniform spaces . . ........... 107

Erwin Kreyszig and John Todd, On the radius of univalence of the function

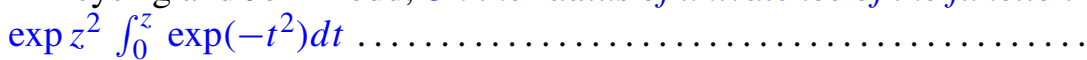

Roger Conant Lyndon, An interpolation theorem in the predicate

calculus......................................... 129

Roger Conant Lyndon, Properties preserved under homomorphism ........ 143

Roger Conant Lyndon, Properties preserved in subdirect products ....... 155

Robert Osserman, A lemma on analytic curves ................ 165

R. S. Phillips, On a theorem due to Sz.-Nagy..................... 169

Richard Scott Pierce, A generalization of atomic Boolean algebras ....... 175

J. B. Roberts, Analytic continuation of meromorphic functions in valued fields................................. 183

Walter Rudin, Idempotent measures on Abelian groups ................ 195

M. Schiffer, Fredholm eigen values of multiply-connected domains ........ 211

V. N. Singh, A note on the computation of Alder's polynomials ......... 271

Maurice Sion, On integration of 1-forms ...................... 277

Elbert A. Walker, Subdirect sums and infinite Abelian groups........... 287

John W. Woll, Homogeneous stochastic processes . .................. 293 